\title{
On the fracture locus of AA7075-T651
}

\author{
M. Fourmeau ${ }^{1,2, a}$, T. Børvik ${ }^{1}$, A. Benallal ${ }^{2}$, and O.S. Hopperstad ${ }^{1}$ \\ ${ }^{1}$ SIMlab, Department of Structural Engineering, NTNU, NO-7491 Trondheim, Norway \\ ${ }^{2}$ LMT-Cachan, 61, avenue du Président Wilson, F-94235 Cachan Cedex, France
}

\begin{abstract}
The overall goal of this work-in-progress is to try to establish the fracture locus (i.e. the equivalent strain to fracture as a function of the stress triaxiality factor) for the high-strength AA7075-T651 aluminium alloy. Experiments on several specimen geometries enabled us to reach stress triaxialities in the range from $\sigma^{*}=0$ for shear tests to $\sigma^{*}=1.4$ for notched specimen tensile tests. For shear tests, failure occurred by strong strain localization. However, local stresses and strains can not be deduced directly from these tests. Consequently, Digital Image Correlation (DIC) was used to extract the local strain fields to failure. In parallel, numerical simulations of the tests were run using the non-linear finite element code LS-DYNA to extract the evolution of the local stress triaxiality during straining. Based on a combination of both experimental and numerical data, the failure locus of the material can be constructed.
\end{abstract}

\section{Introduction}

Due to its high strength-to-density ratio, the aluminium alloy AA7075 in T651 temper is frequently being used in many engineering structures (like aircrafts, spacecrafts, ships and armour applications). Such structures are submitted to complex loadings which may lead to material fracture. Ductile fracture of metals occurs by void nucleation, growth and coalescence, and is strongly affected by the stress triaxiality (see e.g. [1] - [4]). However, under certain loading conditions ductile fracture takes place inside localized shear bands. Such fracture may be difficult to describe and predict owing to the low scale at which large plastic deformations and void growth occur.

Exponential failure curves have been proposed by e.g. Rice \& Tracey [5] and Johnson \& Cook [6], while more recently curves with several branches have been proposed by Bao \& Wierzbicki [7]. However, these have been somewhat criticized for its phenomenological origin, and there are still a lack of understanding of the fracture mechanisms when it comes to low stress triaxiality.

A possible explanation of this behaviour can be found at the microstructure level of alloys like the AA7075. Precipitate free zones weaken the grain boundaries and give the alloys a quasi-brittle tendency depending on loadings. It has already been established that the failure mode could be a combination of cavity growth and shear band localization (Pardoen et al. [8]), but quantitative studies of local strains have not been much investigated so far.

The overall goal of this work is to propose a method using both experimental and numerical data to construct a reliable failure locus. Numerical simulations are used to determine the local value of the stress triaxiality, while DIC measurements are used to validate the simulations at a local scale.

a

e-mail : marion.fourmeau@ntnu.no

This is an Open Access article distributed under the terms of the Creative Commons Attribution-Noncommercial License 3.0, which permits unrestricted use, distribution, and reproduction in any noncommercial medium, provided the original work is properly cited. 


\section{Material tests}

\subsection{Material and specimens}

The AA7075-T651 belongs to the Al-Zn-Mg-Cu series with a chemical composition as given in Table 1. In this study, all specimens are extracted from the same $20 \mathrm{~mm}$ thick plate. Anisotropy is known to be important for this rolled aluminium alloy (see the microstructure and the true stressstrain curves from uniaxial tension tests in Fig. 1). However, in this paper we will only focus on the rolling direction, i.e. the longitudinal axis of all specimens are in the rolling direction of the plate. The effect of material anisotropy on the fracture locus will be studied in further work.

Quasi-static tensile tests on smooth and notched axisymmetric specimens and quasi-static shear tests on butterfly specimens have been conducted at ambient temperature (see Børvik et al. [9]-[10] and Eriksson et al. [11] for specimen geometries). The tensile tests were run at a nominal strain rate of $5 \cdot 10^{-4} \mathrm{~s}^{-1}$, while the shear tests were run at an average strain rate of $2.5 \cdot 10^{-3} \mathrm{~s}^{-1}$ in the shear zone.

Table 1. Nominal chemical composition in wt. \% of AA7075-T651.

\begin{tabular}{|c|c|c|c|c|c|c|c|c|c|}
\hline $\mathbf{A l}$ & $\mathbf{S i}$ & $\mathbf{F e}$ & $\mathbf{C u}$ & $\mathbf{M n}$ & $\mathbf{M g}$ & $\mathbf{C r}$ & $\mathbf{Z n}$ & $\mathbf{T i}$ & Others \\
\hline Rest & 0.06 & 0.19 & 1.3 & 0.04 & 2.4 & 0.19 & 5.7 & 0.08 & 0.15 \\
\hline
\end{tabular}
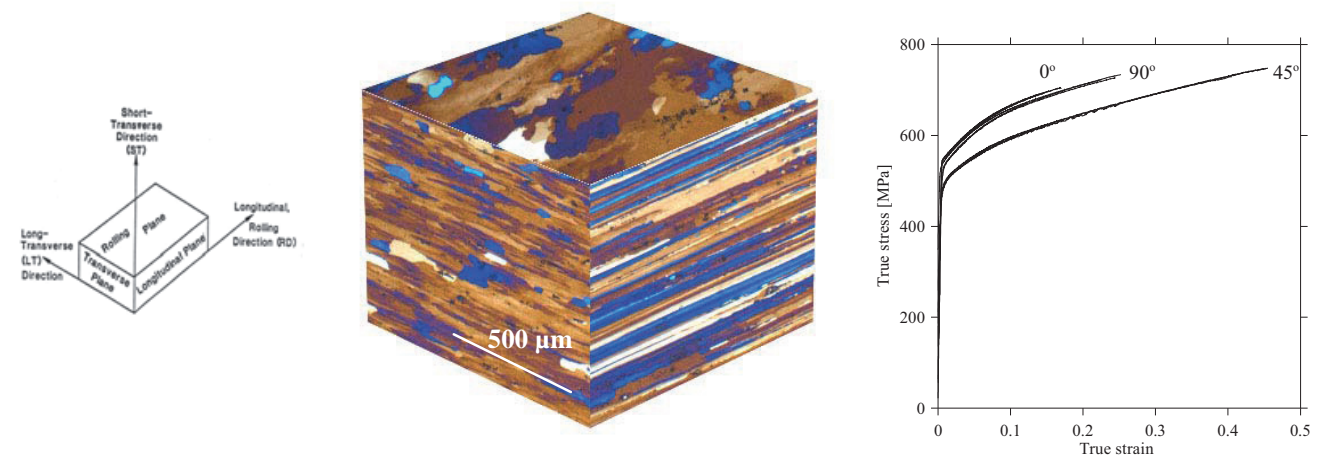

Figure 1. Tri-planar optical micrograph illustrating the grain structure of the AA7075-T651 alloy and true stress-strain curves to fracture in uniaxial tension for three orientations of the specimen with respect to the rolling direction of the plate[10].

\subsection{Experimental set-up}

All material tests were carried out using electro-hydraulic testing machines. Two sets of data were extracted from each test: 1) Global forces and displacements were measured from the test machine. The diameter reduction was also continuously measured for tests on smooth and notched specimens. By measuring the diameter reduction on round specimens during strain hardening, the true stressstrain relation to failure could be obtained. 2) A high-resolution optical camera was used together with Digital Image Correlation (DIC) techniques to register the strain fields for the shear tests.

In the tensile tests, the true failure strain was captured by measuring the diameter reduction. In the shear tests, the specimen's geometry does not enable a direct link of the macroscopic force and displacement to local stress and strain. Thus, DIC measurements were required for the shear tests. 


\subsection{Experimental results}

True stress-strain curves are shown in Fig. 2 (left) for tensile tests on smooth and notched specimens. The expected decrease in strain to failure is observed when the stress triaxiality is increased (i.e. the notch radius is decreased). Force-displacement curves from shear tests are shown in Fig. 2 (right).

\subsubsection{Fracture mechanism}

The macroscopic failure mechanisms are very different when the specimen type is changed. For tensile tests on smooth specimens, necking is not observed and the specimen fractures along a $45^{\circ}$ surface (with respect to the loading direction). For tensile tests on notched specimens, we get a typical cup-and-cone ductile fracture. Void coalescence starts at the centre and the specimen finally fractures along a $45^{\circ}$ surface near the edge of the specimen. For shear tests, the fracture occurs along a flat surface oriented in the loading direction, after relatively large local deformations.

\subsubsection{Strain measurement}

The computation of the local deformation at the location of fracture is different for different test specimens. For tensile tests on round specimens, the local axial strain in the notch is obtained from diameter reduction and by assuming volume conservation. A strong assumption induced is the homogeneity of axial strain in the notch section. This will be discussed later in the paper.

For the shear test, local strains at the fracture location are extracted by DIC using the postprocessor Correli (Besnard et al. [12]. Black dots are painted on the white background of the specimen to obtain a speckle pattern. Planar strain fields are obtained by comparison of two successive pictures of the specimen. Planar strains are presented in Fig. 3. Here 1 and 2 denote the loading and orthogonal directions, respectively, while E11 is the axial strain, E22 is the transverse strain and E12 is the shear strain. The local strain at failure location is extracted from the zone-ofinterest (ZOI) in Fig.3. The shear strain E12 is obviously the largest, but axial and transverse strains are not negligible. This shows that the shear test does not induce a pure shear loading state.

\section{Numerical simulations}

\subsection{Finite element model}

Finite element simulations are run applying the non-linear explicit code LS-DYNA [13] using solid elements with one integration point and stiffness-based hourglass control. Mass-scaling was used to decrease the computation time. The numbers of elements used for the different specimen geometries are summarized in Tab. 3. A modified version of the Johnson-Cook constitutive relation was used to describe the material behaviour. The equivalent stress is expressed as

$$
\sigma_{e q}=\left(A+B \varepsilon_{e q}^{n}\right)\left(1+\dot{\varepsilon}_{e q}^{*}\right)^{C}\left(1-T^{* m}\right)
$$

where $\varepsilon_{e q}$ is the equivalent plastic strain and $A, B, n, C$ and $m$ are material constants. The dimensionless plastic strain rate is given by $\dot{\varepsilon}_{e q}^{*}=\dot{\varepsilon}_{e q} / \dot{\varepsilon}_{0}$, where $\dot{\varepsilon}_{0}$ is a user-defined reference strain rate. The homologous temperature is defined as $T^{*}=\left(T-T_{r}\right) /\left(T_{m}-T_{r}\right)$, where $T$ is the absolute temperature, $T_{r}$ is the room temperature and $T_{m}$ is the melting temperature. Parameters for AA7075-T651 were calibrated from material tests in the $0^{\circ}$ direction, and are given in Table 2 . No fracture criterion was used in the material model. Consequently, simulations are run to higher strains than those observed in the experiments. 
Table 2. Material and physical constants for the AA7075-T651 in $0^{\circ}$ direction [1010].

\begin{tabular}{|c|c|c|c|c|c|c|c|c|c|c|c|c|c|}
\hline $\begin{array}{c}\boldsymbol{E} \\
(\mathrm{GPa})\end{array}$ & $\boldsymbol{v}$ & $\begin{array}{c}\boldsymbol{\rho} \\
\left(\mathrm{kg} / \mathrm{m}^{3}\right)\end{array}$ & $\begin{array}{c}\boldsymbol{A} \\
(\mathrm{MPa})\end{array}$ & $\begin{array}{c}\boldsymbol{B} \\
(\mathrm{MPa})\end{array}$ & $\boldsymbol{n}$ & $\begin{array}{c}\dot{\boldsymbol{\varepsilon}} \\
\left(\mathrm{s}^{-1}\right)\end{array}$ & $\boldsymbol{C}$ & $\begin{array}{c}\boldsymbol{T}_{\boldsymbol{r}} \\
(K)\end{array}$ & $\begin{array}{c}\boldsymbol{T}_{\boldsymbol{m}} \\
(K)\end{array}$ & $\boldsymbol{m}$ & $\begin{array}{c}\boldsymbol{C}_{\boldsymbol{p}} \\
(\mathrm{J} / \mathrm{kg} / \mathrm{K})\end{array}$ & $\boldsymbol{X}$ & $\begin{array}{c}\boldsymbol{\alpha} \\
(/ K)\end{array}$ \\
\hline 70 & 0.3 & 2700 & 520 & 477 & 0.52 & $5 \cdot 10^{-4}$ & 0.001 & 293 & 893 & 1 & 910 & 0.9 & $2.3 \cdot 10^{-5}$ \\
\hline
\end{tabular}

Table 3. Numbers of solid elements for each specimen geometry.

\begin{tabular}{|c|c|c|c|}
\hline Smooth specimen & Notched specimen $\mathbf{R}=\mathbf{0 . 8} \mathbf{~ m m}$ & Notched specimen $\boldsymbol{R}=\mathbf{2} \mathbf{m m}$ & Butterfly specimen \\
\hline 36000 & 43264 & 43136 & 18628 \\
\hline
\end{tabular}

\subsection{Validation of simulations}

Predicted stress-strain curves are found to be in good agreement with the experimental results for tensile test on smooth specimen (see Fig. 2). However, the simulations clearly over-predicted the stress for both the tensile tests on notched specimens and the shear test. This over-prediction was also observed by Bai and Wierzbicki [14], who stated that a possible explanation could be hydrostatic stress sensitivity in the material. Indeed, the hardening law parameters in this study are calibrated from a smooth tensile test. However, if the induced hydrostatic stresses influence the plasticity mechanisms, the used model is not able to predict tests where hydrostatic stresses are predominant. Another observation is that the smooth test curves are very different for different material directions (see Fig. 1), while notched and shear test curves have shown to be more similar with direction. Thus, the effect of anisotropy in the material could be another explanation for this deviation. This is currently being invested, and will not be shown in the present paper.

Despite the over-prediction of the stress-strain curves in the simulations of notched and shear specimens, the triaxiality paths are found to be rather insensitive to the material input. Consequently, the predicted evolution of the stress triaxiality with plastic straining can still be used with confidence in the rest of the study. Moreover, for the shear tests the numerical strains are comparable to DIC measurements in Fig. 3, and the measured and predicted strain components are shown to be in good agreement. Thus, even if the stress-strain curve is over-predicted by the simulations, the local strains in the shear area seem to be well discribed.
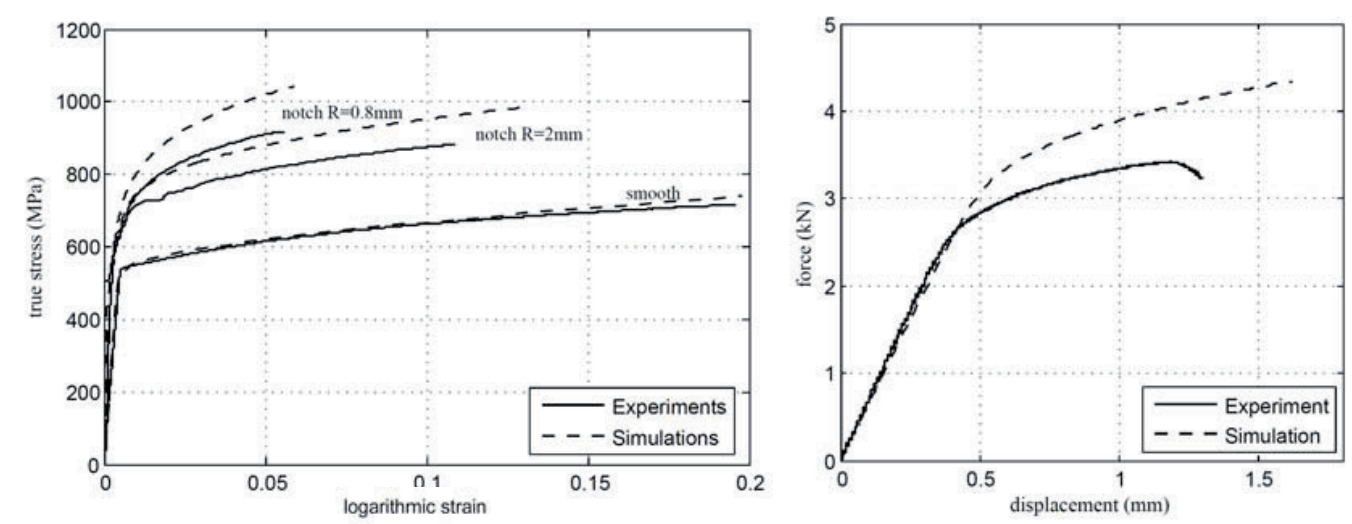

Figure 2. Experimental and numerical stress-strain curves for tensile tests on AA7075-T651. 

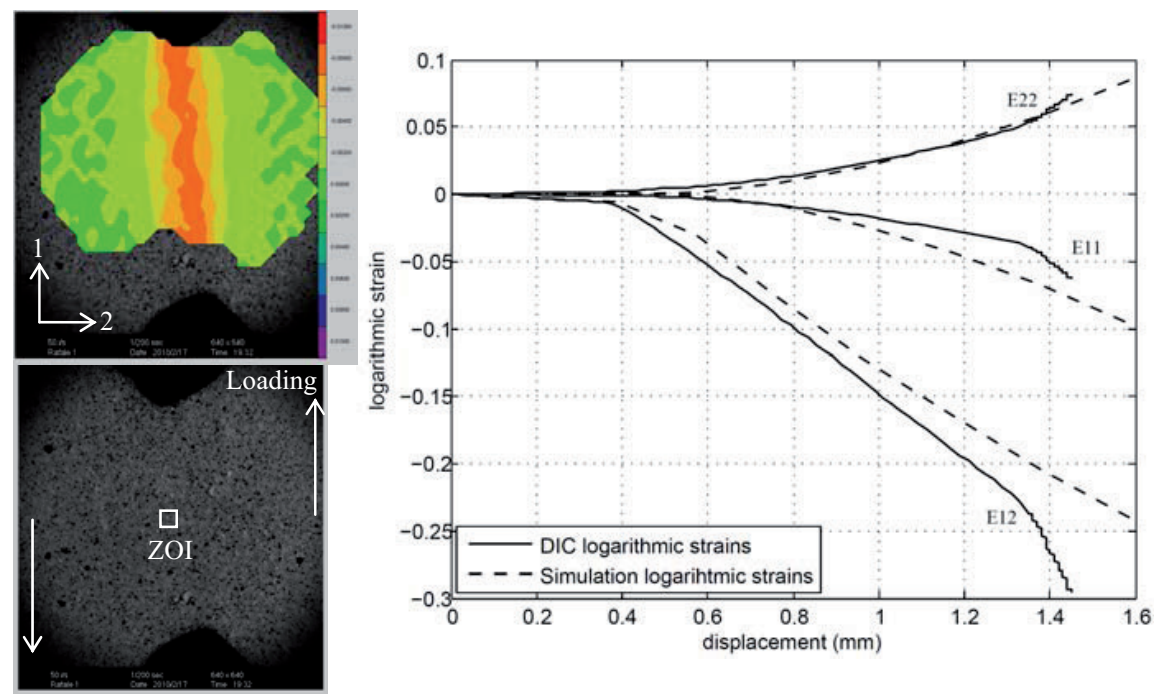

Figure 3. Measured (DIC) and predicted strain components from the shear test on AA7075-T651.

\subsection{Stress triaxiality extraction}

The stress triaxiality is non-uniform and evolves during plastic strain hardening. Indeed, at a local scale, this evolution is important and must be taken into account. The triaxiality is extracted from elements where fracture is assumed. In Fig. 4, stress-triaxiality curves are presented for different elements from the centre to the border of the notched specimen with $\mathrm{R}=2 \mathrm{~mm}$. Different triaxialities and plastic strain paths are seen for different elements. This means that the fracture mechanism involves elements having different loading histories. One may suppose that the fracture process starts in the centre for smooth and pre-notched axisymmetric specimens where the stress triaxiality has a maximum. This was e.g. pointed out by El-Magd [15], who used the evolution of the stress triaxiality from the central element to establish the failure locus. A similar approach is used in the present paper. It should however be noted that for large stress triaxialities, represented by high a/Rratios (where a is the specimen radius and $\mathrm{R}$ is the radius of the notch), the fracture may initiate at the surface of the notch root. Thus, the starting point of failure in notched specimens is first unknown and cannot be located directly (Børvik et al. [9]). This is due to the fact that both the stress triaxiality and the accumulated plastic strain are driving the damage and fracture process.

An inaccuracy in this procedure should also be pointed out. The experimental fracture point obtained for the specimen corresponds to the fracture of the surface. This means that we over-predict the strain to failure of elements in the centre of the specimen. It also means that we do not capture the correct triaxiality path for the surface elements, since fracture is supposed to occur at the centre. However, the fracture process is very fast compared to the whole tension test and the error in triaxiality or in strain to failure would concern only a negligible part of the total path.

A last point to discuss is the value of the equivalent strain at fracture. For e.g. tensile tests on notched specimens $(\mathrm{R}=2 \mathrm{~mm})$, the equivalent plastic strain for different elements scatter between 0.14 and 0.22 , whereas the experimental value calculated from diameter reduction is equal to 0.11 . This shows the limitation of the assumption of strain homogeneity for local strain calculations. This also compels to consider carefully the triaxiality paths, which do not correspond to real specimen states in some cases. 


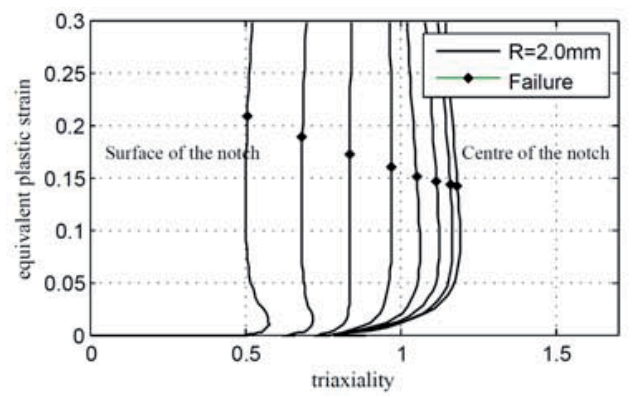

Figure 4. Equivalent plastic strain as a function of stress triaxiality for tensile test on notched specimen, at different points.

\section{Failure curve construction}

In this section, the failure locus is constructed using a combination of both the experimental and numerical data (local equivalent strain and local stress triaxiality paths). This locus will then be compared to the fracture locus constructed based on the initial stress triaxiality.

Fig. 5 shows the triaxiality versus equivalent plastic strain curves (or the fracture locus) for the AA7075-T651. There are several points to notice. First of all, the final difference in failure locus between the one constructed based on the initial stress triaxiality and the one based on simulated stress triaxiality paths is not very large (the latter is slightly higher even though the stress triaxiality paths especially for the notched specimens vary quite considerably during straining). Secondly, the failure locus is rather linear within the range of stress triaxiality investigated. However, negative stress triaxialities (using e.g. upsetting tests) have not been considered in this study. Thirdly, there is no drop in failure strain when going from the smooth tensile test $\left(\sigma^{*}=1 / 3\right)$ to the shear test $\left(\sigma^{*}=0\right)$ for this material. Such a drop has been reported for other alloys by e.g. Bao and Wierzbicki [7]. Here, the local failure strain to failure in the shear test was extracted from DIC measurements.

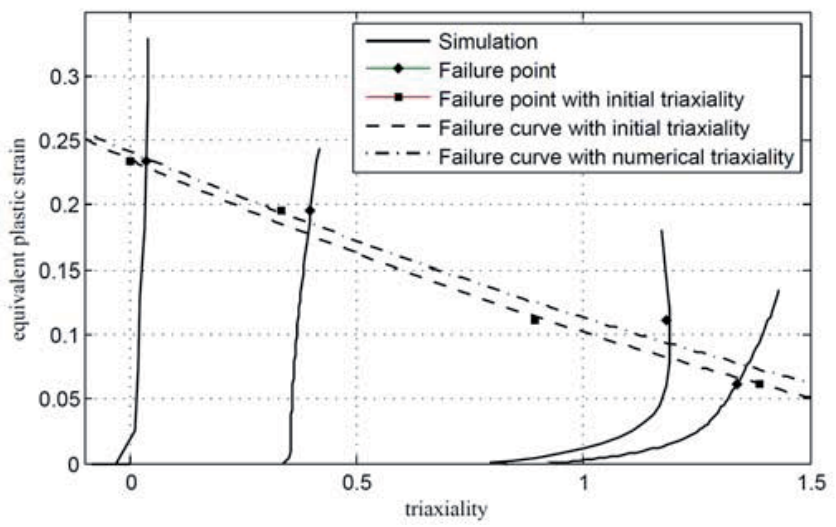

Figure 5. Failure locus constructed with initial stress triaxiality or with predicted stress triaxiality. 


\section{Conclusions and perspectives}

This paper shows the first steps of a method which aims at combining experimental results and numerical simulations to establish a failure locus of a complex material. The study was limited to 4 types of tests and only one direction of the material (even though the material was proven to be very anisotropic both in plastic flow and strain to failure).

One conclusion of the study is that using the predicted triaxiality path instead of the initial one leads to a higher failure locus. This is due to the fact that the stress triaxiality mainly increases during straining. This also means that the approximation of constant initial triaxiality is conservative.

This study raises many important points which have to be investigated further. Firstly, the material model could be enhanced in order to better fit experiments. A material model representing the complex plastic anisotropy of the alloy should be adopted for this purpose. Secondly, for the tensile tests the local strain calculation could be improved in order to validate the numerical simulation more properly, as it was done for the shear test. Thirdly, investigations of the microstructure are required to establish the link between the strain to failure and the micromechanisms leading to strain localisation and failure. Finally, numerical simulations of lower triaxiality tests have to be conducted to establish a failure locus for a larger range of stress triaxialities.

\section{References}

1. F.A. McClintock, Journal of Applied Mechanics 35, 363-71 (1968)

2. A.L. Gurson, Journal of Engineering Material Technology 99, 2-25 (1977)

3. P.F. Thomason, Ductile fracture of metals (Oxford, Pergamon press, 1990)

4. T.L. Anderson, Fracture Mechanics (Fundamentals and Applications). CRC Press (1991)

5. J.R. Rice, D.M. Tracey, Journal of the Mechanics and Physics of Solids 17, 201-217 (1969)

6. G.R. Johnson, W.H. Cook, Int. Jour. of Engineering Fracture Mechanics 21, 31-48 (1985)

7. Y. Bao, T. Wierzbicki, International Journal of Mechanical Sciences 46, 81-98 (2004)

8. T. Pardoen, D. Dumont, A. Deschamps, Y. Brechet, Journal of the Mechanics and Physics of Solids 51, 637-665 (2003)

9. T. Børvik, O.S. Hopperstad, T. Berstad, European J. of Mechanics A/Solids 22, 15-32 (2003)

10. T. Børvik, O.S. Hopperstad, K.O. Pedersen, Int. Jour. of Impact Engineering 37, 537-551 (2010)

11. M. Eriksson, O.-G. Lademo, O.S. Hopperstad, Proceedings of 9th Int. Conf. on Material Forming (ESAFORM-2006), Glasgow, April 26-28, pp. 331-334 (2006)

12. G. Besnard, F. Hild, S. Roux, Experimental Mechanics 46, 789-804 (2006)

13. LSTC, LS-DYNA Keyword User's Manual, Version 971, (Livermore Software Technology Corporation, California, 2007)

14. Y. Bai, T. Wierzbicki, International Journal of Plasticity 24, 1071-1096 (2008)

15. E. El Magd, M. Abouridouane, International Journal Of Impact Engineering 32, 741-758 (2006) 